\title{
Construyendo una Interface para el Ambiente Virtual ROODA - Rede c00perativa de Aprendizagem
}

\author{
Patricia Alejandra Behar \\ Alulsio Pinheiro \\ Juliano de Vargas Bittencourt \\ Silvia de Oliveira Kist
}

\section{Constructing a New Interface for ROODA Environment}


Resumen: El proyecto de investigaciÛn ROODA- Rede cOOperativa De Aprendizagem (en espaÒol: Red Cooperativa de Aprendizaje) - trata de un rea de conocimiento interdisciplinario, que integra la Tecnologia Digital con la EducaciÛn, como forma innovadora de creaciÛn de ambientes virtuales de aprendizaje. El objetivo de este, es la construcciÛn de un ambiente sobre el cual puedan ser realizadas investigaciones y estudios sobre la efectividad de las tecnologias digitales en los cursos de pregrados y postgrados, visando el desarrollo cognitivo, sÛcio-afectivo y profesional de estudiantes y profesores. Para eso fueron utilizados recursos telem ticos con el propÛsito de promover la comunicaciÛn/ interacciÛn/cooperaciÛn dentro del ambiente y las diferentes formas de producciÛn de material a travĖs de la Web. En este paper, particularmente, ser- presentado el estudio que fue desarrollado para la construcciÛn de una nueva interface en la 'Itima versiÛn del ROODA.

Palabras-clave: Aprendizaje virtual. Aprendizaje cooperativo/coleborativo. InteracciÛn hombre-m·quina. Interface centrada en el aprendiz.

\begin{abstract}
This research project ROODA - Cooperative Learning Network - deals with an interdisciplinary area that integrates the Digital Technology and Education, as an innovationtechnological mechanism to createdistance learning environments. The main goal of this project is the development of an environment where a research can be carried out on the effectiveness of the digital technology on the graduate and postgraduate schools. It aims the cognitive, socio-affective and professional developing of the students and professors. Therefore, telematic resources were used to promote the communication/interaction/cooperation inside the environments, and different forms of material production through the Web. In particular, in this paper, it is presented the study of a new interface for the last version of the ROODA environment.
\end{abstract}

Keywords: E-learning. Cooperative/Collaborative learning. Human-computer interaction. User centrated interface.

BEHAR, Patricia Alejandra; PINHEIRO, Alul̀sio; BITTENCOURT, Juliano de Vargas; KIST, Silvia de Oliveira. Construyendo una Interface para el Ambiente Virtual ROODA - Rede cOOperativa de Aprendizagem. Informática na Educação: teoria \& prática, Porto Alegre, v. 6 , n. 1, p. 23-40, jan./jun. 2003. 


\section{Introducción}

El proyecto de investigaciÛn ROODARede cOOperativa De Aprendizagem - (en portugues RODA significa rueda, círculo, dentro de la idea que todo está conectado con todo, no hay un punto de partida ni de llegada; en español: Red Cooperativa de Aprendizaje) aborda un -rea de conocimiento interdisciplinario que integra la Tecnologia Digital con la Educaciûn como forma innovadora de creaciûn de plataformas ${ }^{1}$ de aprendizaje a distancia.

Por lo tanto, el objetivo de este estudio es la contrucciÛn de Plataformas Computacionales que suportem Ambientes de Aprendizaje Construtivistas, que visan (1) al desarrollo de investigaciones y estudios sobre la efectividad de las tecnologias de la informaciÛn y de la comunicaciÛn en los cursos de graduaciÛn y postgrado; (2) al desarrollo cognitivo, sÛcio-afectivo y profesional de estudiantes utilizando estas nuevas tecnologias; (3) promover la formaciûn de recursos humanos en Inform tica Educativa; (4) utilizar recursos telem ticos en el proceso de interacciÛn entre estudiantes, desarrollando experiencias de intercambio/ cooperaciÛn entre ellos; (5) publicar y organizar, en repositÛrios no-lineares e interconectados, la producciÛn de grupos envueltos en este tipo de ambiente - procesos, reflexiones y resultados del trabajo entre alumnos.

Este artìculo presenta una de las partes del proyecto, que es un estudio de interface para el desarrollo de la misma en la 'Itima versiÛn del ambiente ROODA. Por lo tanto, se discute la import,ncia del proyecto de interfaces en plataformas para EAD en general. Luego, ser. presentado el ROODA, su filosofia y funcionalidades, el estudio realizado sobre interfaces, la interface actual, los trabajos futuros y, por 'Itimo, las consideraciones finales. Cabe enfatizar que este proyecto hace parte de los estudios que est $\cdot n$ siendo desarrollados en el NUTED - N'cleo de Tecnologia Digital aplicada a la EducaciÛn, de la Faculdad de EducaciÛn (FACED), que hace parte del CINTED ñ Centro de Estudos Interdisciplin.rios en Nuevas Tecnologias en la EducaciÛn, de la Universidade Federal do Rio Grande do Sul, Porto Alegre, RS, Brasil.

\section{El ambiente ROODA}

En la mayoria de los cursos a distancia, cada alumno recibe o tiene acceso al material sobre algun tema desde su casa, realiza los ejerclccios siguiendo una secuencia linear y evolutiva, y al final del curso hace un examen para garantizar que los contenidos fueron realmente ìmemorizadosî. Se cree que exista una tendencia de las plataformas para EAD en recrear las mismas formas de enseÒanza transmisiva vigentes, cambiando apenas el espacio de las aulas presenciales para la Internet.

Frente a los cambios tecnolÛgicos e pedagÛ́gicos de los 'Itimos tiempos, se sabe que el aprendizaje hoy en dia est - ligado a los conceptos de cooperaciÛn, autoria, individualidad, exploraciÛn, producciÛn, participaciÛn activa del alumno en el proceso, entre otros. Luego, uno de los autores en que se baseÛ este estudio para explicar el apredizaje y aplicar esta idea en la

1 En este estudio la plataforma es entendida como la herramienta en sì y, cuando se refiere al ambiente es considerado la plataforma y sus usu.rios. 
construcciÛn de ambientes virtuales de aprendizaje, es Piaget.

Por lo tanto, Piaget afirma que, para conocer y aprender es preciso actuar sobre el objeto a ser apreendido, interactuar con otros sujetos. Para eso, el aprendiz pasa por un proceso de modificaciÛn estructural produzida por un doble juego de asimilaciÛn y acomodaciÛn (PIAGET, 1970).

En la simple transmisiûn de informaciÛn, como pasa en la mayoria de las aulas, normalmente no hay una acciÛn sobre el objeto, por lo tanto no hay construcciÛn de conocimiento. Dentro de esta visiûn, el alumno es responsable por la construciÛn de su conocimiento, pero el papel de la cooperaciÛn/interacciÛn. y la intervenciÛn del profesor pasan a ser imprescindibles. Por lo tanto, utilizando como base esta idea, el papel del alumno y del profesor cambian considerablemente y, con eso, los propios ambientes y cursos a distancia.

Luego, el papel del profesor es resignificado, o sea, en un ambiente virtual de aprendizaje, este no repasar-informaciÛn, pero si deber- provocar situaciones de desequilìbrio en los alumnos, bien como, la orientaciÛn de su proceso de construcciÛn de conocimiento. El alumno deber-ser participante y activo en su apredizaje.

Es necesario mencionar todavia, que este resalta la importancia que tiene lo social, o sea, las interacciones interindividuales en el desarrollo mental individual que, por su vez, explica el apredizaje. Asi, este es provocado en las perturbaciones decurrentes de la interacciÛn, pudiendo decir que elstas producen una estructura mas compleja construl̀da a travÈs de otras mas simples (MA«ADA, 2001).
EI ROODA es un ambiente virtual de apredizaje cuyo objetivo es el cambio de este paradigma educacional transmisivo y, los conceptos de interacciÛn, cooperaciÛn y comunicaciûn son la llave de este proceso. En este sentido, se desarrollû un ambiente virtual que tiene por objetivo eliminar la distancia entre los participantes, promoviendo formas de ìencuentros virtualesî y constituyendo espacios de convivencia que posibilitan a los aprendices situaciones de conflictos sociocognitivos. Asì, en el momento en que dos o mas indivilduos trabajan en conjunto podr.n ocurrir estos conflictos que los lleven a desequilìbrios y posteriormente a nuevas situaciones de equilìbrio (equilibraciÛn mayorante), en una dialĖctica sin fin (PIAGET, 1970).

En la plataforma ROODA, el aprendiz tiene a su disposiciÛn herramientas de comunicaciÛn sincronicas y asincronicas que tienen como objetivo propiciar el trabajo cooperativo. Adem.s de ello, esta posee algunas herramientas para reflecciÛn personal y reas en donde los profesores disponibilizan el material de los cursos. Estos recursos son agrupados sobre un paradigma centrado en el aprendiz y no en el curso. En el primer caso, el usu.rio entra en el ambiente y tiene acceso a informaciones referentes a todos los cursos en que est $\cdot$ matriculado. Ya, en el segundo, existe una compartimentalizaciÛn por cursos, en donde el alumno tiene que estar siempre dentro del espacio virtual de alguna c·tedra.

Cabe destacar que el ROODA es un software licenciado sobre GPL, baseado en la propuesta de Software Libre. El desarrollo del sistema como Free Software surgî̂ de la filosofia del mismo que, sobre cierto aspecto, defiende ideas muy semejantes a 
algunas lineas de la educaciûn. Ambos defienden una sociedad en que no exista propiedad sobre el conocimiento y donde la competencia sea substitulda por la colaboraciÛn entre las partes. Este aspecto $\mathrm{n}$, o ser abordado en este paper pero se considerÛ importante caracterizar su filosofia.

Actualmente, la cara del ROODA fue remodelada, o sea, se hizo un estudio de interface y esta fue toda redibujada. La nueva versiÛn tiene, como principal objetivo, tornarse mas intuitiva al usu.rio, para que las personas con menos experiencia en el uso de la computadora puedan utilizar el ambiente de la forma mais natural posible. Esta reformulaciÛn se debe a las experiencias y observaciones realizadas con alumnos en interacciÛn con el referido ambiente, en diferentes c.tedras dadas en la Facultad de Educaciûn de la Universidad Federal do Rio Grande do Sul, Porto Alegre, Brasil. En sus relatos ellos apuntaron las dificultades encontradas para lidar con el ambiente y, principalmente con la interface. Estos datos fueron colectados desde el primer cuatrimestre de 2000 hasta el presente periodo de clase. En este paper no ser $\cdot n$ detallados los datos registrados pero, es importante mencionar a tìtulo de informaciûn que hubo este procedimiento para la reformulaciÛn del objeto de estudio del presente abordaje, su interface.

EI ROODA ya se encuentra en su tercer versiÛn, desde su primer implementaciÛn, en el 01/2000 y es utilizado en la prûpia universidad y en otros estados del pals.

\section{Un estudio de interface}

Inicialmente el proyecto ROODA se preocupÛ con cuestiones referentes a la implementaciûn de herramientas que posibilitan la interacciÛn y cooperaciÛn en ambientes virtuales. Adem.s de eso, este tenia que estar consensuado con metodologias pedagûgicas que envuelvan al alumno en su apredizaje activo, participativo con sus compaÒeros, y con el nuevo papel del profesor. No entanto, en el decorrer de las pr.cticas del ambiente con alumnos, surgieron cuestionamientos sobre si las prerrogativas anteriores eran de hecho suficientes para garantizar la efectividad de una plataforma de EAD. A travĖs de la interacciÛn del usu-rio (profesores y alumnos) con el ambiente, se sintiÛ un abismo entre la intenciÛn del grupo de desarrollo (proyectistas y programadores del ROODA) y el uso concreto del ambiente. En muchos momentos, se observÛ que el usu.rio creaba ìfalsos modelosî sobre el funcionamento de una determinada herramienta. Eso lo llevaba a no conseguir realizar la tarea deseada, excluyĖndolo de la colectividad formada por sus colegas. De esta manera, se generaba en el aprendiz y/o profesor un sentimiento de impotencia y/o incompetencia sobre los artefactos tecnolûgicos.

A partir de estos hechos, se percibiÛ que la interface que mediaba la comunicaciÛn entre el hombre y el ordenador, era el punto crìtico del ambiente ROODA. La falla en la interface comprometî̂ otros factores del ambiente, como el planeamiento pedagûgico y las herramientas de interacciûn, que tuvieron que ser reestructuradas, como ser·n presentadas en la secciÛn 4.

Segun Norman (1990) la interface es una antepara que se coloca entre el usu.rio y la tarea a ser ejecutada. En la visiûn de este autor, la interface de un software debe ser invisible, permitiendo al usu-rio concentrar su atenciÛn apenas en la tarea a ser ejecutada. ...l comenta en su obra 
(NORMAN, 1990) que, por ejemplo, ning ' carpintero presta atenciûn en el martillo cuando va a clavar un clavo, su atenciÛn est concentrada sobre la acciÛn a ser ejecutada. En esta analogia, el computador pasa a ser una herramienta para ejecuciÛn de tareas que, en la mayor parte de los casos, requiere mas energia para sì prûpio, que para el objetivo inicial.

Sobre este foco se pueden observar interacciones en cursos a distancia, los cuales en su inicio, muchos mensajes de los alumnos y profesores tratan sobre la versatilidad tecnolÛgica. MaÁada (2001), en su estudio, apuntÛ que los alumnos se atienen mas al domìnio de la herramienta computacional, que al aprendizado de un determinado contenido. Hechos semejantes fueron observados en las experiencias realizadas con el ROODA, en donde el objeto de estudio trataba de un determinado tema y no sobre las herramientas en si.

Entretanto, muchos proyectistas de plataformas para educaciûn a distancia reducen y minimizan este problema al subentender el tÈrmino interface. Muchas veces este tÈrmino es usado para referirse unicamente a los gr.ficos, colores y layout de la pantalla pero, en realidad, comprende todas las acciones envueltas en la interaccion/ comunicaciÛn entre el sujeto y la m·quina. Este desentendimiento puede llegar a traer sĖrias consecuencias a un ambiente virtual de aprendizaje.

Pudo ser constatado, que la mayor parte de los programadores de software retienen su atenciÛn en las propiedades del cÛdigo fuente, tales como clareza, identaciÛn, documentaciÛn, etc; y relegan a un segundo plano la interface. Es muy comun encontrar el desarrollo de proyectos de sistemas enteros sin ninguna consideraciÛn a la interface. No hay motivos para dudar que, cuando se trata del desarrollo de plataformas de EAD, el proceso no sea muy diferente, por lo contrario. Los programadores y analistas de las mismas, creen que la interface es un tema secund.rio para el usu.rio.

Por lo tanto, en los estudios realizados sobre interface se constatû que uno de los aspectos que es preciso llevar en cuenta son los conceptos fundamentales, condiciones e im.genes para que el objeto de interacciÛn seafacilmente comprendido. Esta idea es la que se denomina de met·foras de interface.

Lo importante en una interface es la creaciÛn de objetos de interacciÛn que generen una imagen que sea presentada al usu-rio y con la cual este pueda interactuar. Estos objetos llenan la pantalla de la interface con el usu.rio y pueden basarse en estas met·foras citadas anteriormente, a travÈs de su representaciÛn, sea esta en forma de botones, ventanas, menus, interruptores, entre otros. Luego, la idea es que el aprendiz no necesite ìpensarî sobre el ambiente en sì, concentrandose en el objeto de estudio que, en este caso, seria el contenido especlłfico de una matĖria. O sea, la interface del ambiente no puede constituirse en un problema para el alumno/profesor, pero si en un recurso que sea ìtransparenteíi para ellos.

Una buena interface necesita tener elementos que simplifiquen la realizaciÛn de tareas para que el aprendiz no necesite aprender nuevos comandos o tipos de navegaciÛn (CASSIPANI, 2000). TambiÈn es muy importante que los İconos que aparecen en la pantalla sean conocidos del p' blico, 
intuitivos, para que el usu.rio se sienta a gusto $\mathrm{y}$, adem.s de ello, que permitan asociaciones con el mundo real, basandose en objetos existentes en el cotidiano.

Lo que impulsionÛ el estudio de una nueva interface fue el an-lisis empl̀rico realizado a travÈs de entrevistas y observaciones en clases de pregrado y posgrado, sobre cuestiones que se relacionaban con aspectos de la interface y del ambiente como un todo.

Como consecuencia, se partî̂ para la construcciÛn y estudio de una idea basada en met·foras para el desarrollo de una nueva interface del ROODA. El objetivo, a partir de entonces, fue facilitar la interacciûn y el aprendizaje de los alumnos, o sea, que estos no necesiten de ìentrenamientoî para el uso del ambiente y que consigan trabajar de forma r.pida y eficaz con el mismo.

En el momento de la construcciÛn de una interface, es muy importante tambièn, definir las directrizes para el planeamiento gr.fico que lleve en consideraciÛn preocupaciones relacionadas con la percepciÛn visual del alumno y con la estĖtica. Adem·s de una diagramaciÛn leve y harmonica, que no sobrecargue demasiado la p·gina, se pueden crear Ìconos que representen los diversos momentos y preocupaciones pedagÛgicas contenidas en diversos cursos del ambiente, bien como cuadros e ilustraciones. De esta forma, se crea un vìnculo mas estrecho con el alumno, visando el enriquecimiento tem tico y visual que facilite la interlocuciÛn del material con el alumno. Asi, la mediaciÛn pedagûgica tramita de la satisfacciÛn visual a la apropiaciûn e identificaciÛn del producto por su interlocutor.
Como fue dicho, la ergonomia y el design tienen que ser llevados en cuenta pues el alumno pasar. horas frente a la pantalla del ordenador, conviviendo con colores, formas y muchas informaciones. Para Ios alumnos la estÈtica es una calidad que atrae. La interface de un ambiente tiene que estar bien presentada, con recursos atrayentes, que estimulen la navegaciûn, la descubierta de nuevas posibilidades, que sean intrigantes y que tengan un lenguaje que llame la atenciÛn (CASSIPANI, 2000).

Por lo tanto, se cree que el proceso de desarrollo de cualquier ambiente virtual debe partir del usu-rio y sus necesidades. Como dice Norman, (1998), ìla buena tecnologia es aquella que sirve al usu.rio, las tareas y sus dificultadesî. Luego, el producto centrado en el usu.rio requiere un desarrollo que entienda a las personas y sus actividades di.rias. Para tanto, el primer paso a ser tomado para obtener un buen produto, es la observaciûn del usu.rio frente al ambiente y asì se podr. afirmar que la usabilidad se torna tan importante como el design del sistema.

Llevando en cuenta estos argumentos, se defiende el estudio intensivo de pruebas de interfaces para plataformas de educaciÛn a distancia, durante el prÛpio desarrollo de las mismas, como forma de minimizar el impacto del uso de esta tecnologia en los aprendices. El mÈtodo a ser utilizado debe ser definido en cada proyecto, pues cada plataforma e interface tiene sus particularidades.

Norman (1990) presenta varios ejemplos de frustraciones enfrentadas por las personas que no pueden ejecutar tareas simples del dia-a-dia. Se puede pensar en la 
utilizaciÛn de la computadora como una de esas actividades que traen reacciones emocionales, y muchas veces son marcantes para el sujeto.

Este tipo de experiencia marca la forma como cada persona se relaciona con los artefactos tecnolÛgicos. De la misma forma, se puede decir que las interfaces de plataformas para EAD condicionam la manera que un aprendiz se relacionar. con el curso, su contenido, con los otros sujetos del ambiente y como ser. su forma de educaciÛn a travÈs del mismo. A seguir se transcriben algunas experiencias en donde eso quedÛ evidente.

Por ejemplo, en la segunda versiûn de la plataforma ROODA existia un bug en el programa que exibia en el rea de trabajo del ambiente la frase que decia ìNenhuma mensagem para vocíî ñ en portugues (ìningun mensaje para ustedî). En realidad, este sistema deberia exibir las alteraciones en el ambiente (fÛrum, chat,di-rio de abordo), desde la 'Itima vez que el usu·rio saliû (hizo el logout) del mismo, pero por falta de tiempo en ese momento, no pudo ser bien implementada, dando una idea erronea de lo que habia sucedido.

Durante el uso del ambiente ROODA, v.rios usu-rios reportaron su frustraciûn porque nunca habian recibido ni siquiera un mensaje. En realidad, ellos se sentian exclù̀dos del grupo, como muestra la transcripciÛn del trecho abajo:

$\mathrm{n}, \mathrm{O}$, logo que eu digito o meu user e a minha senha, ele abre j. dizendo que n,o tem nenhuma mensagem para mim. Por que ninguÈm manda mensagem pra mim? :-( (mensaje del Finder capturada de la Base de Datos) ${ }^{2}$
Esta sensaciÛn de inseguridad hacla con que el sujeto piense que estaba siendo exclulddo del grupo, sin recibir ninguna atenciÛn y totalmente aislado. A tìtulo de ilustraciÛn, ser.n presentados algunos ejemplos que demuestran este tipo de sensaciÛn frente al ambiente. De cualquier forma, es importante enfatizar que, a partir de las manifestaciones de los usu.rios no se pudo $\mathrm{m} \cdot \mathrm{s}$ considerar la interface como un objeto tan excento como, normalmente, se pensaba.

Luego, se supone que la interface condiciona la forma como los aprendices van a interactuar dentro del sistema, y consecuentemente, con sus pares. Por lo tanto, es imposible eliminar la importancia que existe en la construcciÛn de una interface, principalmente, en plataformas para EAD.

\subsection{Prerrogativas de una Interface WEB}

Una interface se compone de in 'meros factores, tales como gr.ficos (layout), ergonomicos (colores), funcionales (navegaciÛn dentro de la interface) y subjetivos, por ejemplo, la facilidad de aprendizaje y de ejecuciÛn de tareas, bajo porcentual de errores y satisfacciÛn del usu.rio en la interacciÛn con el ambiente (WINCKLER, 2000). Todos estos aspectos componen la parte estructural de una interface determinando su usabilidad y accesibilidad.

Uno de los mayores problemas de la usabilidad de una interface es la dificuldad del usu.rio en encontrar las informaciones deseadas en una determinada interface. Por lo tanto, para que no ocurra este tipo de cosa, se debe primar por la claridad y objetividad en la creaciÛn de un ambiente. La interface

2 No, luego que yo digito mi user y mi seÒa, el abre ya diciendo que no hay ningun mensaje para mi. Porque nadie me manda mensajes? 
debe permitir que el usu.rio aprenda a utilizar las herramientas, siguiendo una secuencia lÛgica de razonamiento.

En relaciÛn a la accesibilidad, se puede resaltar los softwares empleados en la utilizaciÛn del ambiente: los browsers o navegadores. EI ROODA tiene como meta que su plataforma sea compatible con la mayoria de los browsers existentes (Internet Explorer, Netscape, Opera, Mozilla, etc.). Para eso, fue hecha una investigaciûn para asegurarse que el usu-rio podria utilizar el navegador que est - acostumbrado en su casa o trabajo para interactuar con el ambiente, sin que el mismo necesite instalar plugins, o hasta browsers diferentes.

De acuerdo con las ideas elaboradas anteriormente, se puede afirmar que el design de una interface, sea esta de un simple site o de un ambiente, es una de las partes mas importantes en la construcciûn del mismo, ya que se trata de la parte mas visible al usu.rio.

Por lo tanto, la construcciûn de esta necesita de algunas prerrogativas: debe ser - gil (no llevar mas de 10 segundos para cargar); ser independiente de resoluciÛn o tamaÒo del monitor del usu.rio (llenar la pantalla sin barra de rolaje horizontal); dar libertad de movimiento al usu.rio; $y$, adem.s de todo, tener ideas especlificas sobre su proyecto, sus usu-rios y sus necesidades (NIELSEN, 2000).

Se habla mucho sobre la utilizaciûn de colores en la construcciÛn de sites, como ya fue colocado, pero se argumenta poco sobre ello. En verdad, existe una sÈrie de aspectos importantes en la percepciÛn y utilizaciÛn de determinados colores y tonali- dades en el desarrollo de una p.gina para Web. Seg $n$ estudios realizados sobre el uso de colores en interfaces WWW (BASSO et al, 2000), el proceso de percepciÛn de colores por el hombre influencia en la realizaciÛn de sus tareas.

La tercer versi Ûn del ROODA llevÛ en cuenta los estudios realizados sobre interface y, por lo tanto, una de las alteraciones hechas es sobre el contraste que respeta la ley de percepciÛn del ojo humano, en donde predominan el blanco sobre el azul y el negro sobre el blanco.

Como el ROODA es mas do que un simple site, y si un ambiente virtual de aprendizaje, que puede ser abordado como una simbiosis entre una p.gina y un software, se optÛ por la aplicaciÛn de metaforas en su construcciÛn, Ilevando al usu-rio a la idea de herramientas listas para el uso. En contraste evidente con los colores utilizados, los Ìconos saltan al ser sobrepasados por el mouse, permitiendo que el mismo sepa en donde est clicando y para dÛnde est yendo dentro del ambiente. Este fue un acrÈscimo importante al proyecto de la interface del ROODA, pues esta ofrece sugerencias al aprendiz de como debe proceder una vez que estÈ dentro del ambiente.

El design del ROODA sigue el padrûn de botones del iMac. Fue proyectado e implementado de esta forma, por ser un design moderno, limpio e que resalta a los ojos. Permite tambiÈn una claridad visual sin poluciÛn, llevando el usu·rio directo al contenido deseado.

En consecuencia de este layout, la disposiciûn del contenido propiamente dicho obtuvo un espacio bastante amplio en la 
p·gina, permitiendo una gran visualizaciûn que se adecua de acuerdo con la densidad de informaciones. La parte textual del ambiente es dirigida a los fûrums, chats, datos personales y $\mathrm{p} \cdot$ ginas publicadas. El texto es simple, directo y objetivo, proporcionando rapidez y claridad en la b' squedad de algo.

Se debe resaltar, que el ambiente del ROODA fue desarrollado a partir del usu rio y totalmente direccionado para el. Por lo tanto, las contribuciones de los usu.rios que lo utilizan en sus c tedras o el mal funcionamento/desempeÒo de alguna herramienta, hace con que el grupo de desarrollo y mantenimiento del mismo lo adapte y reformule frequentemente. Por ese motivo, el ROODA est en constante an-lisis, reformulaciÛn y desarrollo.

\section{La interface actual}

A partir de los estudios realizados sobre interface, se sintiÛ necesidad de reestructurar la misma y consecuentemente, el ambiente como un todo. La interacciÛn del aprendiz con el ambiente estaba siendo perjudicada al punto de una subutilizaciÛn de determinadas herramientas debido a una interface poco intuitiva y nada ilustrativa.

La tercer versî̂n del ROODA fue utilizada en cuatro c·tedras con alumnos de pregrado y posgrado de la UFRGS. Estos levantaron v-rios problemas de interface del ambiente a travÈs de sus anotaciones en el fÛrum y di-rio de abordo. El registro de estos datos sirvieron de base para la construcciûn de la nueva interface, como ya fue colocado anteriormente. A seguir son descriptas algunas de sus funcionalidades que fueron modificadas en consecuencia del estudio realizado sobre interface.

\subsection{Navegando en el Ambiente}

Partiendo de las experiencias directas con los usu.rios del ROODA a travĖs de la observaciÛn y entrevistas, se vislumbrû la posibilidad de mejorar la parte visual e interactiva del ambiente. Iniciando por la parte organizacional del ambiente, fue destacada una barra superior en donde fueron disponibilizadas las herramientas de comunicaciÛn (fûrums, chat y e-mail). La idea beneficia la interface y permite que el usu.rio no se pierda dentro del ambiente, permaneciendo visible durante todo el tiempo de navegaciÛn (NIELSEN, 2000).

La p·gina inicial del ROODA, llamada de Webfûlio, permite al aprendiz accesar el conjunto de sus producciones y contribuciones en el ambiente. Dentro del WebfÛlio, existe un rea de trabajo que posibilita la verificaciûn de la caja de correo, mensajes de fûrums, p·gina publicada, datos personales, di·rio, clases y producciones. Adem.s de esto, el usu.rio tiene informaciones y acceso a lo que fue realizado por los grupos de trabajo durante su ausencia en el ambiente.

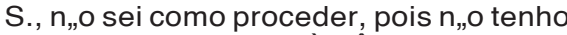
acesso as aulas. n,o È sÛ entrar na pag do rooda que eu consigo ver as aulas? tentei falar com a P. ou a SK. mas elas nao est, o la. o que fazer?† (mensaje del Finder $)^{3}$

Cabe destacar que no ser-n utilizados los nombres de los usu.rios para mantener su anonimato, por esa razÛn, solo fueron usadas abreviaturas.

3 ìS., no se como proceder, pues no tengo acceso a las clases aulas. No es sÛlo entrar en la pag del rooda y consigo verlas? IntentÈ hablar con a P. o con SK. pero ellas no est·n all·. Que hago?î 


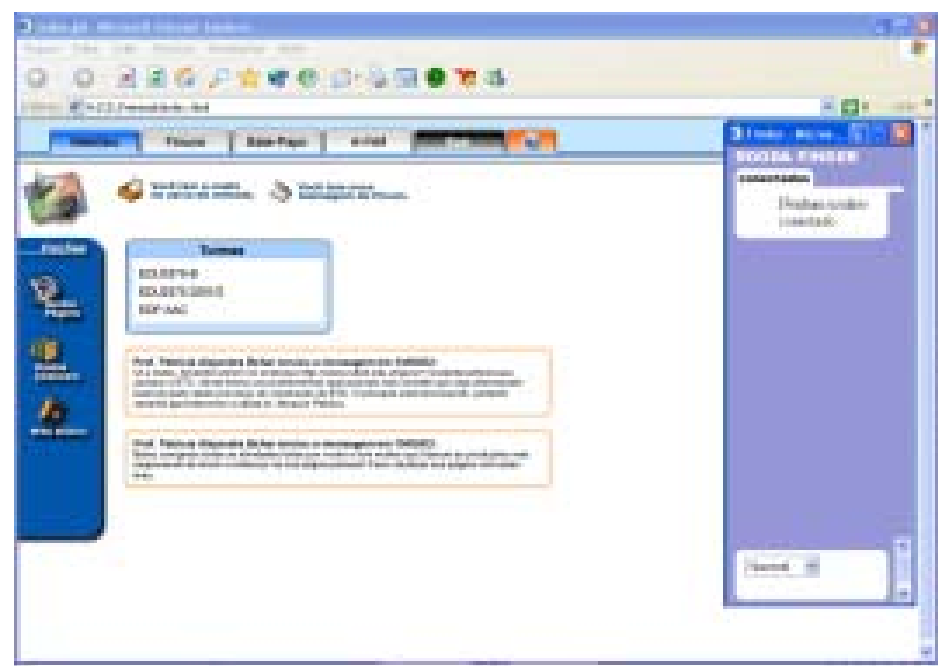

IlustraciÛn 1 - jrea de Trabajo de la Tercer VersiÛn del ROODA

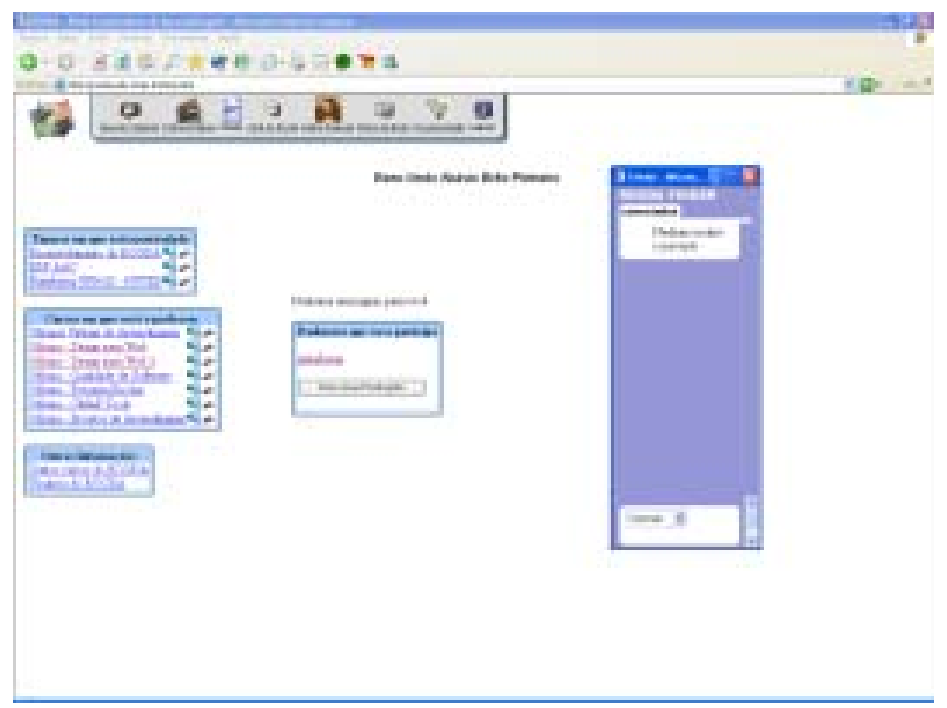

IlustraciÛn 2 - ¡rea de Trabajo Remodelada

Observando el mensaje de arriba, se nota que la alumna no podia encontrar el material de las clases dentro del ambiente. Eso sucediû porque, para accesar la p.gina de la c.tedra, era neces.rio primeramente entrar en la clase, y despuÈs elegir la opciÛn de visualizar la p·gina de la misma. Fue constatado que se trataba de una acciÛn muy simple pero resultaba en un camino un poco largo, con v.rios cliques y, que los usu.rios se perdian. Por lo tanto, en la nueva versiûn, fue incluldo un atajo que posibilita al usu.rio accesar directamente la p.gina. 
... eu achei uma coisa bem legal pra colocar na lista (sobre o brasil exportar tecnologia em EAD), mas nao to achando um lugar que trate da disciplina no geral.... tem alguma dica??? ;) (mensaje del Finder $)^{4}$

Senti falta de um lugar na lista que trate a disciplina como um todo, sem direcionar para uma das aulas. Algo como a ìsala do recreioî, para dar dicas mais abrangentes. S.† (mensaje del Di·rio de Abordo) ${ }^{5}$

En los trechos descriptos arriba, se puede observar la incomprensiÛn, por parte de los usu.rios, de como realizar determinadas tareas. Las funcionalidades que los estudiantes piden a travÈs de sus manifestaciones, en realidad existen en el ambiente, pero son resignificadas e interpretadas por los aprendices de otras maneras. Para resolver algunos de esos problemas, fue creada una herramienta Ilamada agenda. Esta es activada en el momento que el alumno entra en el espacio de la c·tedra, para que estos puedan visualizar, en un primer plano, las informaciones importantes descriptas en ella. Esta herramienta puede ser utilizada para que el profesor coloque mensajes referentes a los aprendices de todo un curso.

\subsection{Producciones}

Es un sistema que posibilita el trabajo cooperativo entre los aprendices de una clase. Permite a los alumnos compartir archivos (p.ginas, html, texto etc...) y disponibilizar trabajos de manera que estos puedan ser accesados por el equipo de trabajo, compaÒeros y por el profesor. Esto permite que los profesores acompaÒen el desarrollo de los trabajos e intervengan cuando juzguen neces rio. Permite tambièn que los interesados se puedan integrar a los equipos, adem.s de contribuir con comen$\mathrm{t}$-rios y crìticas sobre las producciones. La principal diferencia en la interface actual es el design y la presentaciÛn directa de mensajes del equipo y del sistema, sobre su producciÛn. El objetivo es mantener el usu·rio y/o los visitantes actualizados sobre las modificaciones en sus actividades.

El principal problema encontrado en este sistema fue la falta de un mecanismo en donde el aprendiz pudiera acompaÒar y negociar las actividades que su grupo de trabajo desempeÒa en una producciÛn compartida. A pesar de la herramienta poseer un di.rio de actividades, en donde los alumnos deberian registar el desarrollo de la producciÛn, ellos terminaban por articular su proyecto fuera del ambiente, a travĖs de email o telÈfono. De esta manera quedÛ comprometido el registro del proceso de aprendizaje a travÈs del ambiente, restando, muchas veces, como forma de evaluaciÛn el producto final publicado.

\footnotetext{
Oi SK., vou fazer o trabalho com a D. e talvez a Z. participe conosco. Ontem, por telefone combinamos quais os softwares que iremos analisar ${ }^{6}$.i (mensaje del Finder)

Vamos tentar esquematizar o trabalho, levar a sua apreciaÁ,,o e dividir o trabalho, para j- possamos ir produzindo alguma coisa durante a semana. Qual seu telefone $?^{7}$ (mensaje del Finder)
}

4 ìyo encontrÈ una cosa muy buena para colocar en la lista (sobre Brasil exportar tecnologia en EAD), pero no estoy encontrando un lugar de la c.tedra que tenga una parte general .... tenes alguna sugerencia???)

5 ìSenti falta de un lugar en la lista que trate la c·tedra como un todo, sin direccionar para una de las clases. Algo como el ìp.tio del recreoî, para dar sugerencias mas abrangentes. S.î† (mensaje del Di·rio de Abordo)

6 Hola SK., voy a hacer el trabajo con D. y tal vez Z. participe con nosotros. Ayer, por telefone arreglamos cuales son los softwares que iremos analizar.

7 Vamos intentar esquematizar el trabajo, llevar su apreciaciÛn y dividir el trabajo, para que ya podamos ir produciendo algo durante la semana. Cual es tu telËfono? 
Por lo tanto, la principal alteraciÛn sobre la interface de esta herramienta fue colocar esta funcionalidad en un lugar de destaque, tornandola mas visible. Se piensa que habiendo en la p·gina inicial de la producciÛn un resumen de las "Itimas alteraciones que hubo en la producciÛn y un espacio para negociaciones entre los participantes de la misma, la cooperaciûn entre los usu·rios es favorecida, y aumentan las interacciones a travÈs de la plataforma. Adem.s de eso, el desarrollo de un sistema que mantenga el histÛrico de los archivos enviados favorece a la reconstrucciÛn del camino del aprendizaje.

\subsection{Upload de Archivos}

En realidad, el sistema de upload de archivos no se constituye en una herramienta por sì sola, pero si se trata de una funcionalidad utilizada en v rias otras herramientas del sistema para enviar archivos de la m.quina local, en donde el aprendiz trabaja, para la plataformaenla Internet. Por ejemplo, las producciones utilizan el upload para enviar las p.ginas trabajadas por el grupo. La p.gina personal de cada aprendiz es enviada utilizando, tambièn, ese sistema y, los sites de las c·tedras son publicados de la misma manera.

Cuando fue construl̀do el sistema de upload se siguiÛ el padrûn utilizado por otros sites que disponibilizan espacios para publicaciÛn de p.gina como el Yahoo, Geocites y el HP. En este modelo, el usu-rio visualiza los archivos que est. $n$ en el servidor, y puede realizar operaciones sobre estos comocopiar, pegar, borrar, mover, etc. Cuando desea enviar un archivo actualizado, se elige la opciÛn ìenviar archivosî, se selecciona y se clica en enviar.

Entretanto, mismo habiendo sido construldo con base en sistemas de publicaciÛn de p.ginas tan largamente utilizadas, la herramienta de upload del ROODA presentÛ graves problemas de interface. Los usu.rios, principalmente los novatos, tuvieron dificultades en comprender la necesidad de enviar los archivos para el servidor y presentaban errores provenientes de problemas en la construcciÛn de las p·ginas WEB, como referenciar un imagen como archivo local. Algunos de esos problemas no son directamente relacionados al sistema de upload, pero estos no pueden ser ignorados y colocados a cargo de la incapacidad del aprendiz, principalmente cuando se refiere a un ambiente que pretende ser utilizado por personas leigas en tĖrminos de inform·tica.

Hoje, eu e A., tentamos publicar a p.gina, mas $n$, ,o conseguimos. A professora $B$. È que acabou publicando, ficamos atenta para aprender, mas, muitas vezes, fiquei perdida com tanta movimentaÁ,o para a publicaÁ,o. No final, acho que entendi, mas preciso mexer sozinha para ver se realmente entendi publicar uma apresentaÁ,,o powerpoint no rooda. ${ }^{8}$ (mensaje del di.rio de abordo)

Hoje foi um pouco complicado para conseguirmos publicar a p.gina, fiquei confusa. ${ }^{9}$ (mensaje del di.rio de abordo)

Depois de muito tentar conseguimos com a ajuda da professora publicar o trabalho, achei meio complicado todo este movimento para a publicaÁ,,${ }^{10}$ (mensaje del di·rio de abordo)

8 ìHoy, yo y A., intentamos publicar la p·gina, pero no conseguimos. La profesora B. La terminÛ publicando, quedĖ atenta para aprender, pero, muchas veces, me perdi con tantos movimientos para la publicaciÛn. Al final, creo que entendì, pero preciso hacerlo sola para ver si realmente entendi como publicar una presentaciÛn powerpoint en el roodaî.

9 ìHoy fue un poco complicado para que consegamos publicar la p·gina, quedÈ confusa.í

10 ìDespuĖs de intentar bastante consigamos, con la ayuda de la profesora, publicar el trabajo, me pareciÛ medio complicado todo este movimento para la publicaciûnî. 
Estou quebrando a cabeÁa para conseguir publicar, mas $n, 0$ consigo. Os link n,o estou conseguindo fazer, aquele da p.gina inteira. O trabalho $\mathrm{j} \cdot$ est com fundo e digitado no frontpage, $n$,o consegui colocar par o Rooda. ${ }^{11}$ (mensaje del di $\cdot$ rio de abordo)

Como puede ser observado en los trechos arriba descriptos, el principal problema es la incompreensiÛn por parte del usu·rio del modelo de funcionamento del upload. No existe una orientaciÛn clara que le diga al usu.rio como iniciar el proceso, y tampoco una guia que lo ayude durante el mismo. La interface actual destaca el botÛn de enviar de manera a dejar claro para el aprendiz que aquel es, normalmente, el primer paso a ser ejecutado. Una vez que el proceso se haya iniciado, un asistente guia el usu.rio en todos los pasos hasta que la tarea haya sido finalizada.

TambÈm fue creado un filtro, que analiza los archivos enviados, principalmente los HTML en busca de errores comunes, que vienen siendo observados en diversas oficinas de construcciÛn de $p \cdot g i n a s$ realizadas a travÈs del ROODA. Ese filtro avisa al usu-rio del error cometido y le pregunta si, mismo asi, desea enviar el archivo. Adem.s de eso, caso el usu.rio no comprenda el error que cometî̂, una ayuda puede ser disparada. Este sistema visa auxiliar monitores y profesores de cursos a distancia a corregir in 'meras veces los mismos problemas para diferentes personas.

\subsection{Finder}

Se trata de un sistema de mensajes instant·neos que integra todos los apren- dices conectados al ambiente, desde que esten online. Esta herramienta permite que las clases sean dadas fuera del ambiente de aula. El Finder es una ventana a parte en el ambiente que lista todos los usu.rios conectados en el ROODA y posibilita la comunicaciÛn en tiempo real con cada integrante (uno-a-uno).

Estatl̀sticas sobre la base de datos del ambiente ROODA muestran que esta herramienta fue la mas utilizada por los usu-rios. No entanto, su interface tambièn apuntaba graves problemas, algunos de los cuales son presentados a seguir:

Perdi a ultima mensagem. Repete. ${ }^{12}$ (mensaje del Finder)

-†Boa noite, S.! Bom trabalho.†ó fala SK.

-†oi.... Boa noite... mas quem mandou essa msg??? :)†ó responde S. ${ }^{13}$ (di·logo en el finder)

Los mensajes presentados arriba, fueron retirados de la base de datos del finder y apuntan el error encontrado en el modelo de enviar y recibir mensajes, en donde todas las veces que el aprendiz recibiû un nuevo mensaje, una caja de di·logo surgl̀a avis.ndole el nombre del remitente, y pregunt·ndole si deseaba leer la misma. Caso afirmativo, el mensaje era exibido. Entretanto ocurrieron algunos problemas como el de perder mensajes, al no existir un registro de los di·logos anteriores.

Una de las modificaciones realizadas en el ambiente, fue que la conversaciûn en la actual versiÛn, funciona como en un chat,

11 'Me estoy rompiendo la cabeza para conseguir publicar, pero no consigo. Los link no los estoy pudiendo hacer, aquellos de la p·gina entera. El trabajo ya est con fondo y digitado en el frontpage, no consegui colocarlo en el Roodaî.

12 ìPerdì el ultimo mensaje. Repetimeî.

13 - †Buenas noches, S.! Buen trabajo.†ó habla SK.

-†Hola.... Buenas noches... pero quien mandÛ este mensaje??? 
en donde los mensajes son postados en orden de emisiÛn y no se borran m.s a cada nuevo mensaje recebido o enviado. Otra modificaciÛn fue la inclusiÛn de opciones de status (normal, invisible y ocupado). Eso fue neces -rio para posibilitar que los usu rios utilicen el ambiente sin ser importunados, y cuando desearen, alteran su opciÛn de status.

\subsection{Bate-papo}

El Bate-papo del ROODA, surgiÛ en la primera versiÛn del ambiente con el nombre de Chat, denominaciÛn esta que lo acompaÒû hasta este 'Itimo remodelaje del ambiente. Este fue el primer error de interface encontrado en relaciÛn a esta herramienta, el uso del tÈrmino Chat. Esta palabra, es muy significativa para las personas que utilizan Internet hace un tiempo, no entanto no es muy representativa para el usu-rio inexperiente. Por lo tanto, se ha colocado un tËrmino usual en portugues que se refiere a una conversaciÛn informal, el bate-papo.

Sobre esta herramienta fueron realizados muchos estudios por el equipo del ROODA. Muchas plataformas para EAD optan por utilizar pequeÒos applets java que se comunican con un servidor IRC, lo que garantiza una buena performance al sistema. Entretanto, el tiempo requerido para efectuar el download del applet, a travĖs de lineas discadas, es alto. Adem.s de eso, obliga el usu.rio a poseer una JVM (Java Virtual Machine) instalada en su computador.
Otra soluciÛn normalmente utilizada, es forzar lap-gina aque automaticamente realice un refresh (recarga) de si misma, mostrando, asì, los nuevos mensajes en la prÛxima exibiciÛn. Ese fue el abordaje inicial dado al Bate-Papo del ROODA. Entretanto surgieron algunos problemas, como los que son transcriptos abajo, retirados de un fûrum que trataba sobre evaluaciÛn de la plataforma.

A janela do chat, cada vez que d refresh, volta para o inlcio e o usu-rio tem que usar as barras de rolagem, logo n,o vemos online as msgs que est,,o sendo recebidas, alĖm de ficar muito difl̀cl de ler e acompanhar o chat. ${ }^{14}$

$\mathrm{Na}$ janela do chat o bug atrapalhou um pouco, pois È desgastante ficar usando a barra de rolagem. ${ }^{15}$

quanto ao chat: como os colegas j disseram anteriormente o fato de a cada nova mensagem termos que movimentar a barra de rolagem em muitos momentos interferiu no acompanhamento das discussıes. $\mathrm{N}$,o sei se era um problema sÛ do meu micro, mas $\mathrm{n}, \mathrm{o}$ consegui direcionar especificamente para alguns colegas meus coment·rios (aparecia para todos). ${ }^{16}$

Observando estos hechos, el equipo del ROODA buscÛ alternativas tecnolÛgicas que pudieran corregir esta situaciÛn. Fue encontrada una tĖcnica que mantiene una conexiÛn abierta entre el servidor WEB y el browser del usu.rio, que permite al sistema de Chat enviar los nuevos mensajes en el momento que estos llegan a la Base de Datos del ambiente, evitando asi el tiempo de refresh. Otra cuestiÛn bastante trabajada fueron los cÛdigos javascript que controlan

14 La ventana del chat, cada vez que d. un refresh, vuelve para el inl̀cio y el usu.rio tiene que usar las barras de rolage, luego no vemos on-line los msgs que estan siendo recibidos, y queda muy dificil de leer y acompaÒar el chat. 15 En la ventana del chat el bug nos confundiÛ un poco, pues es desgastante estar usando la barra de rolage.í 16 En relaciÛn al chat: como los compaÒeros ya dijeron anteriormente, el fato de que a cada nuevo mensaje tener que mover la barra de rolage en muchos momentos interfirî̂ en el acompaÒamiento de las discusiones. No sÈ si era un problema solo de mi ordenador , pero no pude direccionar especificamente para algunos colegas mis coment·rios (aparecia para todos) 
el rolage de la pantalla. Esos cÛdigos fueron reconstruldos y probados con v-rios browser con el objetivo de mantener la compatibilidad del ROODA con los diferentes navegadores. Ese esfuerzo generÛ un sistema de Chat mucho $\mathrm{m} \cdot \mathrm{s}$ robusto y que fue incorporado al ambiente en su tercer versiûn, generando resultados satisfactÛrios. En las 'Itimas experiencias utilizando la 'Itima versiûn, no fue registrado ningun tipo de reclamo por parte de los usu-rios en relaciûn a esta herramienta.

\subsection{Otras herramientas}

Adem.s de las herramientas presentadas anteriormente, la plataforma ROODA posee otras que no fueron abordadas en este paper por no tratar del foco central del mismo, y porque no sufrieron alteraciones significativas en su interface. La descripciÛn mas detallada de todas las funcionalidades puede ser vista en (BEHAR, 2001).

\section{Trabajos futuros}

Es neces.rio destacar que una interface no se debe restringir a los gustos y preferencias de su autor, pues esta es proyectada para sus usu.rios. Por lo tanto, si esta tiene un proyecto de mala calidad, o un design que no lleve en cuenta su representaciÛn mental, el ambiente ser- sub-utilizado en sus funcionalidades o, simplemente deja de ser usado.

Dentro de esta perspectiva queda claro que el mejor camino para el madurecer de una interface, es probarla junto a sus usu.rios.

Luego, como prÛximo paso de este estudio y, consecuentemente reestructuraciÛn de la interface del ROODA se pretende realizar pruebas empìricas, mas especifica- mente ensayos de interacciÛn (RUBIN, 1991), con el objetivo de validar las alteraciones descriptas durante este paper, y detectar cuales son los puntos que exigen un estudio mas profundo.

En el prÛximo semestre, ser·n dadas tres c·tedras, una en la graduaciÛn del Curso de Pedagogia de la UFRGS y las otras dos en cursos de posgrado, mas especificamente, en la maestria del Programa de PÛsGraduaciÛn en EducaciÛn (PPGEdu/UFRGS) y doctorado del Programa de PÛs-GraduaciÛn en Inform tica en la Educaciûn (PPGIE/ UFRGS). Durante estos experimentos, se desea obtener datos para realizar el an-lisis de la nueva interface del ROODA.

\section{Consideraciones finales}

Se puede concluir que las observaciones directas de los usu.rios con el ambiente sirvieron para llamar la atenciÛn del grupo de design y programaciûn del ROODA que habia llegado el momento de hacer una reestructuraciÛn en la interface. Para ello, fue neces-rio hacer una investigaciÛn profunda sobre interfaces de EAD para adaptar las ideas a la referida plataforma.

Por lo tanto, a travÈs de este trabajo se destaca la importancia del estudio de interfaces en plataformas para EAD que, normalmente, es tratado apenas en el nivel estÈtico siendo ignorados aspectos mas importantes como el impacto emocional que ellas causan en los aprendices e, consecuentemente en su apredizaje. A travÈs de este estudio se mostrû como la interface no puede ser vista como un objeto excento en el proceso de enseÒanza y aprendizaje, y que esta debe ser consistente con el design metodolÛgico elegido para el ambiente. 
El desarrollo de interfaces coherentes no puede ser una preocupaciûn apenas de los programadores de sistemas, pero si debe abarcar todos los responsables por los aspectos pedagû́gicos y metodolÛgicos del mismo.

La secciÛn 4 ha mostrado varios puntos del ambiente ROODA que debieron ser modificados en funciûn de las experiencias generadas con los usu-rios, apuntando la interface como uno de los elementos claves para el aprendizaje del estudiante en ambientes de EAD. Por esa razÛn, fue necesario realizar un estudio profundo sobre este tema para afirmar las cualidades de un producto.

Por lo tanto, la interface actual es el resultado de una investigaciûn realizada por el equipo del ROODA sobre los datos registrados por los usu-rios en el ambiente. Cabe enfatizar que podrian haberse hecho otras ìlecturasîsobre los datos para la resoluciÛn de las mismas cuestiones. O sea, se trata de un estudio especìfico y de un camino encontrado para el design de interfaces, no negando la soluciÛn de algunos problemas detectados y del aparecimiento de otros. Se debe considerar como una obra abierta a v·rias lecturas, y por esa razÛn su desarrollo precisa ser un proceso iterativo, en el cual cada ciclo representa un estado mas refinado que el anterior.

Se puede concluir, que la interface no puede ser encarada apenas como un conjunto de İconos y ventanas de un software o p.gina; esta tiene que ser proyectada y analisada en relaciûn a la interacciÛn existente entre el hombre y la m·quina.

En suma, fue desarrollada una nueva interface en la tercer versiÛn del ROODA que comporta una sÈrie de prerrogativas descriptas en este abordaje y que fueron llevadas en cuenta para tornarla lo $\mathrm{m} \cdot \mathrm{s}$ intuitiva, amigable y de $f \cdot c i l$ acceso y navegaciÛn para sus usu.rios.

Por fin, es importante destacar el trabajo de investigaciÛn, creaciûn, implementaciÛn y aplicaciÛn de todo un ambiente de EAD en diversos cursos de la universidad y su gran aceptabilidad por parte del p' blico de alumnos y profesores.

\section{ReferÍncias}

BEHAR, P.; KIST, S.; BITTENCOURT, J. A Caminho de um Ambiente para a EducaÁ,o a Dist,ncia: Rooda - Rede Cooperativa de Aprendizagem. In: SIMP"SIO BRASILEIRO DE INFORM EDUCA « $\mathrm{V}, 12 ., 2001$, s.I. Anais... S.I.: SBC, 2001. P. 89-95.

BORGES, R.; WINCKLER, M.; BASSO, K.. ConsideraÁres Sobre o Uso de Cores em Interfaces www. In: WORKSHOP SOBRE FATORES HUMANOS EM SISTEMAS COMPUTACIONAIS, 3., 2000, Gramado. Anais... Gramado: SBC, 2000.

PIMENTA, M. Muitas Faces em Interfaces. In: SIMP"SIO BRASILEIRO DE INFORMiTICA NA EDUCA « $\sqrt{O}$, 11, 2000, Gramado. Anais... Gramado: SBC, 2000. P. 43

CAPISANI, D. (org.). Educação e Arte no Mundo Digital. Campo Grande, MS: AEAD/UFMS, 2000.

JEFRIES et al. User Interface Evaluation in the Real World: a comparison of four techniques. In:

Human Factors in Computing Systems Conference Proceedings on Reaching Through

Technology. S.I.: ACM Press, 1991. P. 119. 
MA«ADA, D. L. Rede Virtual de Aprendizagem: interaÁ„,o em uma ecologia digital. 2001. Tese (Programa de PÛs-GraduaÁ,,o em Inform·tica na EducaÁ,,o) - Universidade Federal do Rio Grande do Sul, Porto Alegre, 2001.

NIELSEN, J. Projetando Websites. S.I.:Editora Campus, 2000.

NORMAN, D. The Design of Everyday Things. Currency/Doubleday, S.I:Mar. 1990.

The Art of Human-Computer Interface Design: chapter why interfaces donít work. S.I.: Addison Wesley Publisher, 1990. P. 209-219.

The Invisible Computer. S.I.: MIT Press, 1999.

PIAGET, J. Psicologia e Pedagogia: a resposta do grande psicÛlogo aos problemas do ensino. Rio de Janeiro: Editora Forense, 1970.

RUBIN, J. Handbook of Usability Testing: how to plan, design and conduct efective tests, wiley \& sons. New York: s.e., 1994.

SANTOS, M. Educação e Arte no Mundo Digital: criarmeu website. Campo Grande:UFMS/CEAD, 2000.

WINKLER, M. AvaliaÁ,,o de usabilidade de sites web. In: WORKSHOP SOBRE FATORES HUMANOS EM SISTEMAS COMPUTACIONAIS, 4., 2001, FlorianÛpolis. Anais... FlorianÛpolis: SBC, 2001.

Aceito para publicaÁ,,o em 2003/1

\author{
Patricia Alejandra Behar \\ Professora adjunta da Faculdade de EducaÁ,,o e dos \\ Programas de PÛs-GraduaÁ,o em EducaÁ,o e \\ Inform·tica na EducaÁ,,o, mestre e doutora em CiÍncia \\ da ComputaÁ,„o/UFRGS. Coordenadora do N`cleo de \\ Tecnologia Digital aplicada ‡ EducaÁ,o. \\ E-mail:pbehar@terra.com.br \\ http://www.nuted.edu.ufrgs.br
}

\title{
Juliano de Vargas Bittencourt
}

Bacharel em Inform tica/UFRGS, mestrando em Educa-

Á,,o PPGEdu/FACED/UFRGS.

E-mail: juliano@edu.ufrgs.br

Silvia de Oliveira Kist

Pedagoga/FACED/UFRGS, bolsista de ITI/CNPq.

E-mail: silvia@faced.edu.ufrgs.br

\section{Alulsio Pinheiro}

Jornalista/PUCRS, bolsista do CNPq.

E-mail: alupinhiero@hotmail.com 